\title{
Investigation of Layer Composition and Morphology in Perpendicular Magnetic Tunnel Junctions
}

\author{
Danielle Reifsnyder Hickey ${ }^{1}$, Hamid Almasi ${ }^{2}$, Weigang Wang ${ }^{2}$ and K. Andre Mkhoyan ${ }^{1}$ \\ 1. Department of Chemical Engineering and Materials Science, University of Minnesota, Minneapolis, \\ MN, United States \\ 2. Department of Physics, University of Arizona, Tucson, AZ, United States
}

As traditional complementary metal-oxide semiconductor (CMOS) technology approaches its limit, alternative technologies such as magnetic tunnel junctions (MTJs) are being explored to replace CMOSbased devices for memory and logic applications. MTJs have advantages such as nonvolatility, low power consumption, and high densities [1]. These features have enabled application in technologies such as magnetic random access memory (MRAM), static random access memory (SRAM), and spin-transfer torque MTJs (STT-MTJs).

MTJs harness spin-dependent tunneling through a tunnel barrier (e.g., $\mathrm{Al}_{2} \mathrm{O}_{3}$ or $\mathrm{MgO}$ ) that is placed between ferromagnetic electrodes. MgO-based perpendicular MTJs (p-MTJs) also exhibit perpendicular magnetization, which enables high density and scalability [2]. Three application criteria for p-MTJs are thermal stability and high values of tunneling magnetoresistance (TMR) and perpendicular magnetic anisotropy (PMA), and thus significant research has been devoted to improving these parameters.

A strategy to increase the TMR and PMA of $\mathrm{CoFeB} / \mathrm{MgO} / \mathrm{CoFeB} \mathrm{p}-\mathrm{MTJ}$ is to incorporate various heavy metals as capping and buffer layers. Tantalum has been widely used, but recently, other metals such as hafnium [3] and molybdenum [4] have been reported. We recently demonstrated that p-MTJs with Mo retain high values of TMR and PMA after annealing at $400^{\circ} \mathrm{C}$, in contrast to their Ta analogues [5]. However, the structural basis for this performance has been unknown.

Here, we present scanning transmission electron microscopy (STEM), energy-dispersive X-ray spectroscopy (EDX), and electron energy-loss spectroscopy (EELS) data that characterizes the various layers in Mo- and Ta-based p-MTJs. Several features of interest are elemental mixing between layers, crystallinity, and interfacial roughness. Figure 1 shows characterization of cross sections of unannealed and annealed Ta samples. Figures 1(a,b) show high-angle annular dark-field (HAADF) and bright-field (BF) STEM images, respectively, of an unannealed Ta-based p-MTJ, and Figure 1(c) shows HAADFSTEM and EDX data displaying the elemental compositions of the layers of an annealed Ta-based MTJ. The data presented here provides insights into how the sample microstructure is related to device performance. STEM imaging and STEM-EDX experiments were conducted using an aberrationcorrected FEI Titan G2 60-300 (S)TEM equipped with Super-X EDX and Gatan Enfinium ER spectrometers, operated at $200 \mathrm{kV}$ [6]. 
References:

[1] P Choudhary et al., IRJET 2 (2015) p. 1635.

[2] S Ikeda et al., Nat. Mater. 9 (2010) p. 721.

[3] T Liu et al., AIP Adv. 2 (2012) 032151.

[4] T Liu et al., Sci. Rep. 4 (2014) 5895.

[5] H Almasi et al., Appl. Phys. Lett. 106 (2015) 182406.

[6] The authors gratefully acknowledge funding provided by C-SPIN, one of six centers of STARnet, a Semiconductor Research Corporation program, sponsored by MARCO and DARPA.
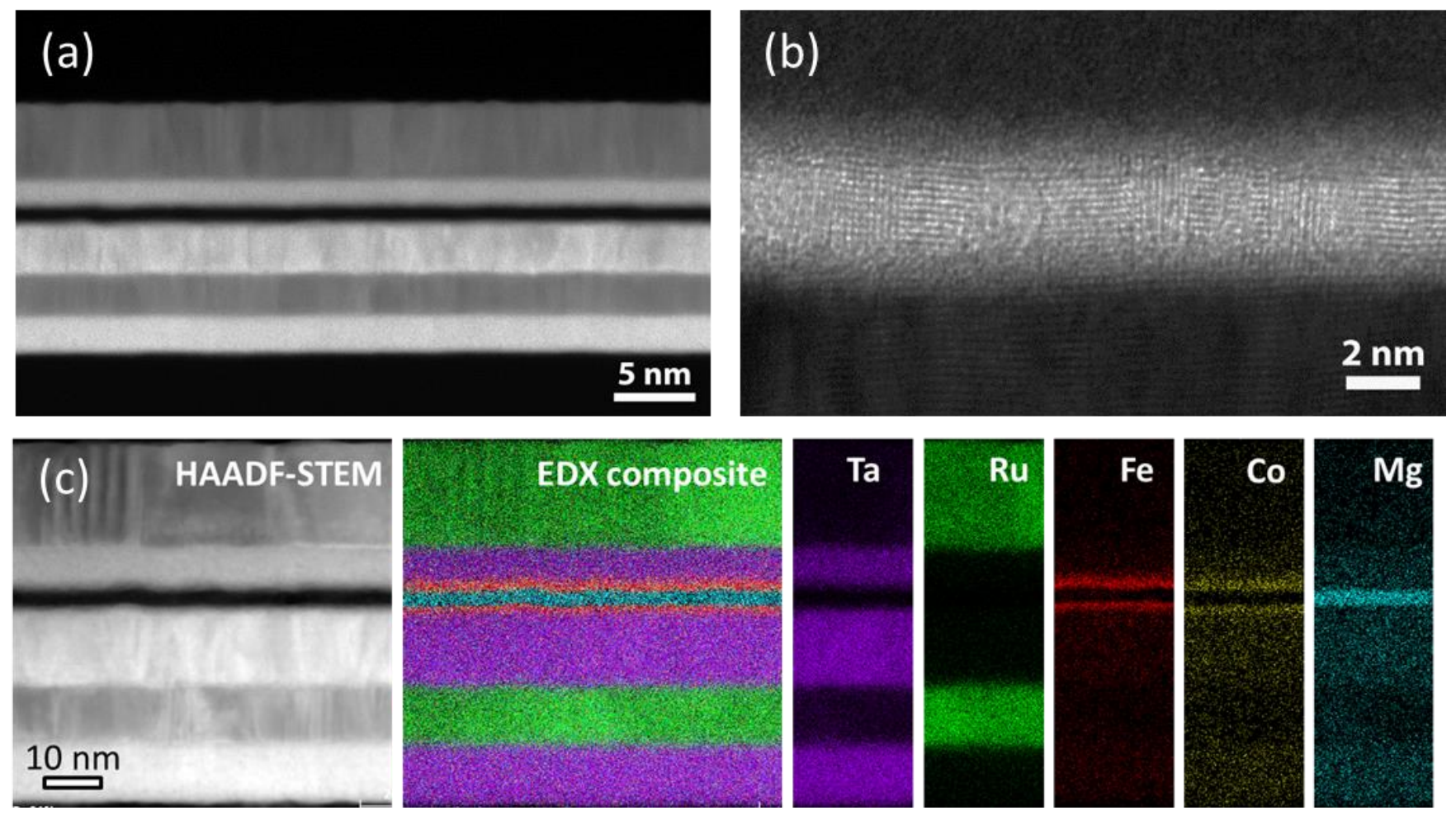

Figure 1. Characterization of Ta-based MTJs. (a) HAADF-STEM image and (b) BF-STEM image of an unannealed Ta-based sample. (c) HAADF-STEM image and EDX maps of an annealed Ta-based sample. 